\title{
Automatic Identification of Large Fragments in a Pile of Broken Rock using a Time-of-Flight Camera
}

\author{
Christopher McKinnon and Joshua A. Marshall, Senior Member, IEEE
}

\begin{abstract}
This paper presents a solution to part of the problem of making robotic or semi-robotic digging equipment less dependant on human supervision. A method is described for identifying rocks of a certain size that may affect digging efficiency or require special handling. The process involves three main steps. First, by using range and intensity data from a time-of-flight (TOF) camera, a feature descriptor is used to rank points and separate regions surrounding high scoring points. This allows a wide range of rocks to be recognized because features can represent a whole or just part of a rock. Second, these points are filtered to extract only points thought to belong to the large object. Finally, a check is carried out to verify that the resultant point cloud actually represents a rock. Results are presented from field testing on piles of fragmented rock.
\end{abstract}

Note to Practitioners-This paper presents an algorithm to identify large boulders in a pile of broken rock as a step towards an autonomous mining dig planner. In mining, piles of broken rock can contain large fragments that may need to be specially handled. To assess rock piles for excavation, we make use of a TOF camera that does not rely on external lighting to generate a point cloud of the rock pile. We then segment large boulders from its surface by using a novel feature descriptor and distinguish between real and false boulder candidates. Preliminary field experiments show promising results with the algorithm performing nearly as well as human test subjects.

Index Terms-Mining and construction automation, field robotics, object identification, point cloud data processing

\section{INTRODUCTION}

$\mathbf{T}$ HIS PAPER is about the problem of automating the digging operations of loading machines used in mining and construction. In a typical underground mining operation rock is broken so that it may be collected at a draw point. It is very difficult to achieve perfect blasting results [1] and in some mining methods there is little to no blasting, which results in less control over the size of the fragments in the rock pile. The end result is that a rock pile can contain fragments of many different shapes and sizes [1]. If fragments are large enough, they must be specially handled to avoid damage to equipment and interruptions to production [1], [2], [3].

For example, some strategies for efficiently excavating piles of earth (not broken rock) require individual piles of material to be handled as distinct objects, where optimal digging is organized around the efficient prioritization of these piles [4]. In mining, large rocks may need to be handled the same

C. McKinnon is with the Institute for Aerospace Studies, University of Toronto, 4925 Dufferin Street, Toronto, ON M5S 2E4 Canada email: chris.mckinnon@mail.utoronto.ca.

J. A. Marshall is with the Mining Systems Laboratory, Queen's University, Kingston, ON K7L 3N6 Canada email: joshua.marshall@queensu.ca.

Manuscript revised February 19, 2014. way for an efficient mining dig planner. If digging is to be automated-which is the premise of this paper-it is therefore important that the autonomous digging machine be capable of automatically recognizing such rocks. This paper presents a method for automatically identifying rocks of a certain size that may affect digging efficiency or require special handling.

\section{A. Problem Background}

A notable amount of work has been done on the hauling and dumping stages for underground mining machines (e.g., for a review, see [5] and references therein). However, the autonomous excavation problem, especially in fragmented rock, remains a much less developed area [6], [7]. In mining, the unique challenge of handling large rocks in a rock pile is twofold. First, rocks can have many different shapes and sizes. Second, the structure and composition of rock piles can vary significantly, both locally and from mine to mine. This paper focuses on the dig planning subproblem, and not machine control; for control, see [7] and references therein.

Current mining practice relies on human operators to manually load excavator buckets. The operator often has a poor view of the pile, especially when the operator works by remote control or through a teleoperator's console. A notable amount of work has been done on autonomous digging in earth or other fine homogeneous material [8], but these do not address the issue of large rocks. One experiment [9], digging in gravel, used a simple $2.5 \mathrm{D}$ occupancy grid where the size of the grid cells has no relation to particles in the rock pile. This was sufficient for digging in fine gravel but may pose problems if large rocks are encountered. Others have built on this work for applications in homogenous materials [6]. For a different application, [10] used a CCD camera and a laser pointed to detect boulders. Another, targeted specifically at excavation for load-haul-dump (LHD) machines, made an effort to avoid large fragments by focusing on the gradient and overall shape of the pile rather than its composition [11].

More recent work in [12] used the normal-distributions transform (NDT) to classify 3D LiDAR data as planar or uneven. Uneven surfaces indicated regions likely to contain boulders, and planar surfaces could represent tunnel surface (e.g. wall or ceiling) or rock pile depending on their orientation. Although very relevant, this work did not segment out individual rocks, which may be important to achieve efficient automated digging.

\section{B. Identifying Large Objects}

Methods for extracting features from a scene usually make assumptions that allow them to identify the scene's back- 
ground. For example, the "flatland assumption" identifies the ground plane [13] or ground texture, which is used in rover obstacle identification [14]. The background is often the most common occurrence of some measure in a scene. The remaining data is then broken up into independent objects by using edge detection. Others have used surface and surface normal discontinuities to seed points for region selection using deformable solids [15]. Others still have attempted to model rocks with some primitive, such as an ellipsoid [16]. In medical applications, where the number of objects in an image is known, $k$-means clustering has been used [17].

In automated sieving, the whole pile is of interest [18], [19]. Sieving algorithms are usually restricted to more controlled environments, such as over a conveyor; e.g., [3] attempted to segment all rocks on the surface, filtering sets of points that do not represent rocks by a number-of-points criteria.

The authors of [1] tackled a problem preliminary to the one studied in this paper. Their work involved the positioning of a rock breaker above oversized rocks on a grate by using $3 \mathrm{D}$ data from a laser range finder. It was assumed that rocks are overall convex and compact and they used negative curvature minima and occlusions as edges. Edges and discontinuities were connected to form closed convex shapes and superquadrics were used to model rocks. The problem described in this paper is different because a rock pile is naturally much more complex than a simple planar surface in its structure and composition.

\section{Contribution of this Paper}

This paper presents a simple yet robust method for detecting large rocks that does not make the assumption that the surface of the pile is planar and where the number of large rocks is only upper bounded. The focus of this paper is to present a way to segment only large objects (called oversize) on the surface of a rock pile in conditions similar to those present in underground mines. A novel feature descriptor is used to characterize the surface instead of a primitive shape and then the distribution of this descriptor is used to distinguish between real and false large object candidates. This method was tested on piles of broken limestone from road construction; see Fig. 7. Although the method described does not require that the rock pile be of a certain size, piles were chosen from real environments (rather than using laboratory-scale environments).

Like Cheung [1], our approach assumes that rocks are generally convex and closed, however we do not assume that they rest on a flat surface. Faces of large rocks are assumed to be isolated from one another and have at least one feature exposed; i.e., they are not embedded having only one flat face flush with the surface of the pile. These assumptions are not unreasonable for any algorithm hoping to solve this problem by using surface structure alone for automatic analysis.

Conditions underground are often poorly lit and dust and moisture levels can vary significantly. Previous research using digital images noted a dependence on a good viewing angle [2] and lighting [3]. The work presented in this paper used a MESA SwissRanger SR4030 time-of-flight (TOF) camera (see Section III-A). The active TOF camera was found to be well suited for this application because it does not need a

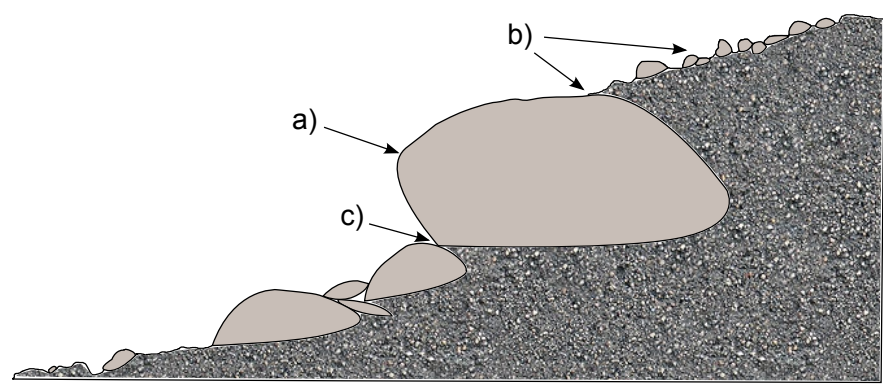

Fig. 1. A 2D illustration of the main types of features present in a rock pile: a) large convex region receives a high score, b) region roughly planar with small surface features receives a score close to zero, and c) large concave region a large negative score. The greyed portion under the rocks is unknown (and not relevant because no part of it can be sensed).

light source and has greater accuracy compared to a stereo camera. The additional surface texture and detailed intensity information provided by a camera has also been found useful for identifying small rocks in desert environments [14], which is different from our objective.

\section{Measure of Curvature}

Like [1], we rely on a variant of curvature to characterize shapes in the rock pile. However, by using the method shown in this paper, round shapes with large volume (rather than sharp outcrops or edges) stand out. This aligns with our goal of finding large boulders rather than edges between rocks (see also the discussion in Section I-B).

Once the user specifies a characteristic feature size, which may be related to the maximum size that equipment can deal with, curvature on that scale can be calculated. Let $\mathcal{P}_{i}$ indicate the set of points within distance $r_{f}$ of point $P_{i}$. We then estimate the center of a region as the centroid of points $\mathcal{P}_{i}$. Let $\mathbf{V}_{c, j}$ be the vector between the centroid $C_{i}$ and point $P_{j} \in \mathcal{P}_{i}$. Let $\mathbf{N}_{j}$ be the surface normal at $P_{j}$. The sum of the cosine of the angle between $\mathbf{V}_{c, j}$ and $\mathbf{N}_{j}$ over all $P_{j} \in \mathcal{P}_{i}$ may be used as a measure of curvature. Each point in a region can contribute from -1.0 to 1.0 to the score of the point at the center of that region. This method is outlined in Algorithm 1, where $S_{i}$ denotes the total "oversize score" at $P_{i}$.

Algorithm 1 Method for scoring points. Fig. 1 shows the three main types of features captured by this method.

$$
\begin{aligned}
& r_{f} \leftarrow \text { user specified feature size } \\
& \text { for (every point } i \text { ) do } \\
& \mathcal{P}_{i} \leftarrow\left\{P_{j}:\left\|P_{j}-P_{i}\right\| \leq r_{f}\right\} \\
& C_{i} \leftarrow \text { centroid of } \mathcal{P}_{i} \\
& \text { for } j=1 \text { to }\left|\mathcal{P}_{i}\right| \text { do } \\
& \mathbf{N}_{j} \leftarrow \text { surface normal at } P_{j} \\
& \quad \mathbf{V}_{c, j} \leftarrow P_{j}-C_{i} \\
& \text { end for } \\
& S_{i} \leftarrow \sum_{P_{j} \in \mathcal{P}_{i}} \frac{\mathbf{V}_{c, j} \cdot \mathbf{N}_{j}}{\left\|\mathbf{V}_{c, j}\right\|}
\end{aligned}
$$

end for

Scaling the region over which curvature is evaluated allows the algorithm to pick up features over a desired range of sizes. 
The highest scoring point will be at the center of a roughly hemispherical feature with no occlusions that is approximately the same size as the user selected scale. This allows for the maximum number of points with normals pointing away from the centroid of the region. A region with a smaller feature (small rock) will not have as many points with normals pointing away from the centroid, and a slightly larger feature (mound on the pile) will likely not have normals as well aligned with $\mathbf{V}_{c, j}$. Occlusions reduce the number of points that are available to contribute to a score.

What makes this method robust for detecting large objects is that features can represent a whole or part of a large object, and features of a range of sizes can receive a relatively high score. These features can be identified, thus allowing the rest of a large object to be recognized at later stages. This is also robust to sensor noise which does not usually have high enough point density or consistency to contribute to a high score since normals pointing in random directions do not consistently increase or decrease the overall score. Similarly, roughness on a scale much smaller than the selected feature size contributes little to the score because positive and negative contributions from small features cancel each other out, resulting in a score that reflects the average profile of the surface.

\section{Method And Example Results}

Fig. 2 shows an overview of the methodology. The following subsections refer to the steps listed in Fig. 2. The algorithms are explained together with example experimental results.

\section{A. Test Sensor and Computing}

The results presented here are based on data collected using an IP67-rated MESA Imaging SR4030 TOF camera, offering a $43.6^{\circ} \times 34.6^{\circ}$ field of view with $0.24^{\circ}$ angular resolution (central pixels) and a range of up to $10 \mathrm{~m}$. Scene illumination is provided by the camera via an array of modulated infrared LEDs. A CCD camera is then used to process the returned light from the environment. To estimate range, the SR 4030 measures the phase difference between the modulated LED signal when it leaves the camera and the light that is sensed by the camera when it returns [20]. For our experiments, all data was collected after sundown. Recall that our target application is underground mines, where there is no ambient light.

The sensor was used via the Robot Operating System (ROS) (Electric) to gather data using the libmesasr Intel driver version 1.0.14-653. The raw point clouds contained 25344 points and included intensity. Fig. 3(a) shows an original point cloud before processing, colored based on returned intensity.

Although benchmarking tests were not performed as part of this research, all of the algorithms discussed in this paper were comfortably run on a Lenovo ThinkPad T420s with Intel i5 processor and 4 GB of DDR3 memory.

\section{B. Step 1: Pre-process and Filter}

The raw point cloud from the TOF camera still contains noise that we do not wish to process. Fig. 4 shows a histogram of intensity for a sample point cloud from our MESA SR4030.

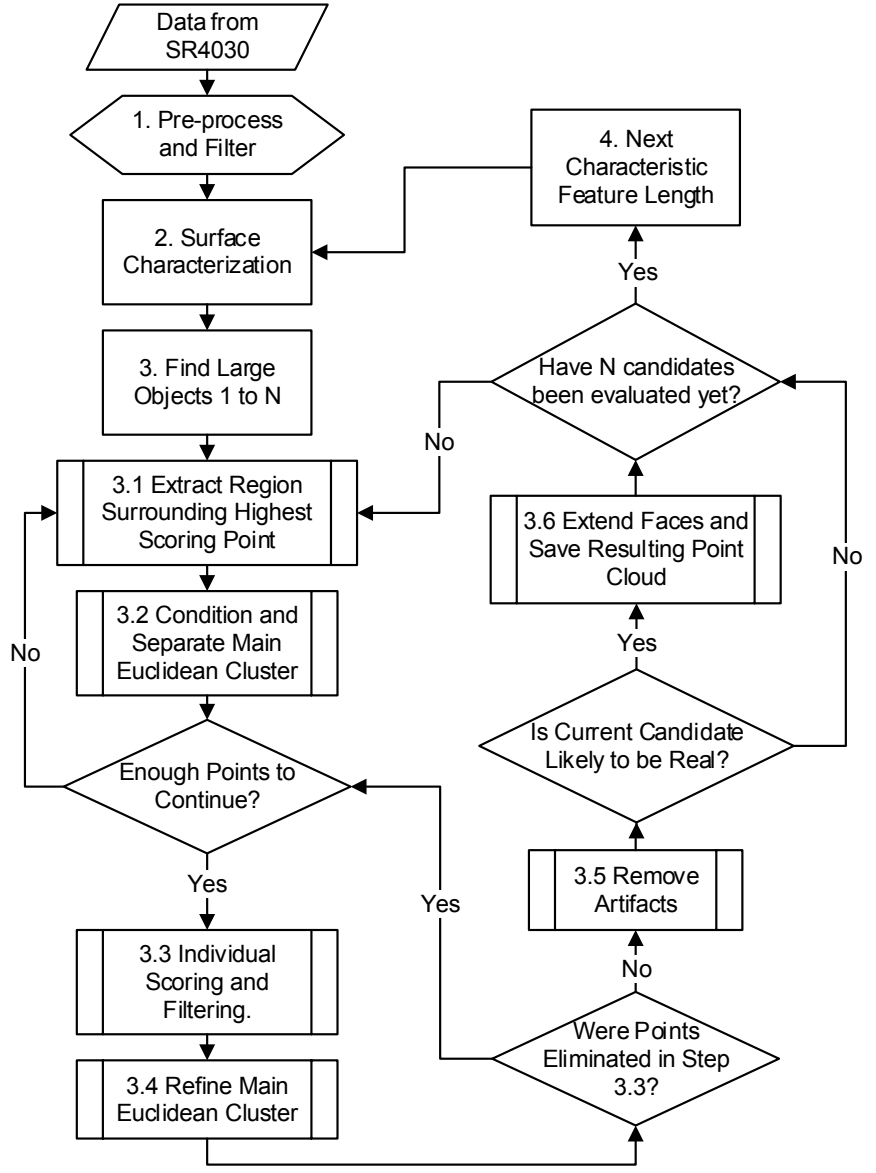

Fig. 2. An overview of the general methodology. The process is terminated at Step 4 if there are no further characteristic feature lengths to be tested.

Points with intensity values below 500 make up the noise in the foreground as well as some of the scatter in the background, which is shown in Fig. 3a by the red points. This allows them to be removed with a simple intensity threshold filter.

Most underground mine tunnels are between 5 and $10 \mathrm{~m}$ wide. Thus, at the ranges needed for our application, there is still too much information for our purposes so we down sample by using a voxel grid ${ }^{1}$. In our experiments, these voxels were square and occupied a space of $0.01 \times 0.01 \mathrm{~m}$. The voxel grid replaces points within the voxel with a single point at the centroid of those points. Cubic voxels are used because this problem requires spatial information in all directions.

Tangent planes are fit to each point with at least five neighbors to characterize the orientation of the surface. Neighbors are points within in a region much smaller than any feature we are interested $i^{2}$; a sphere of radius $0.1 \mathrm{~m}$ was used for this study. The normal to each tangent plane is used as the surface normal at that point. Normals are oriented towards the view point ${ }^{3}$.

The data is down sampled again by using average values of points within the voxel. A cube with edges $0.03 \mathrm{~m}$ long was used for the results presented in this paper. This filter

\footnotetext{
${ }^{1}$ http://pointclouds.org/documentation/tutorials/voxel_grid.php

${ }^{2} \mathrm{http} / / /$ pointclouds.org/documentation/tutorials/remove_outliers.php

${ }^{3} \mathrm{http}: / /$ pointclouds.org/documentation/tutorials/normal_estimation.php
} 


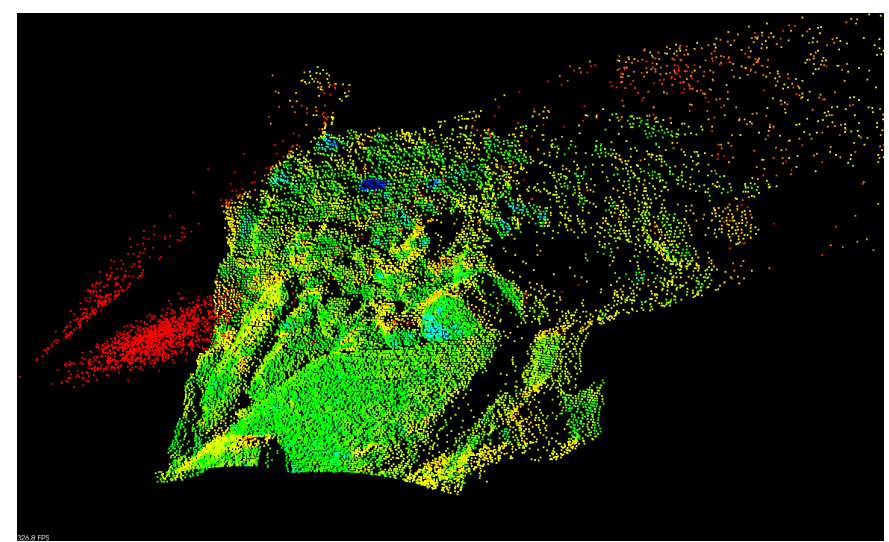

(a) Original point cloud.

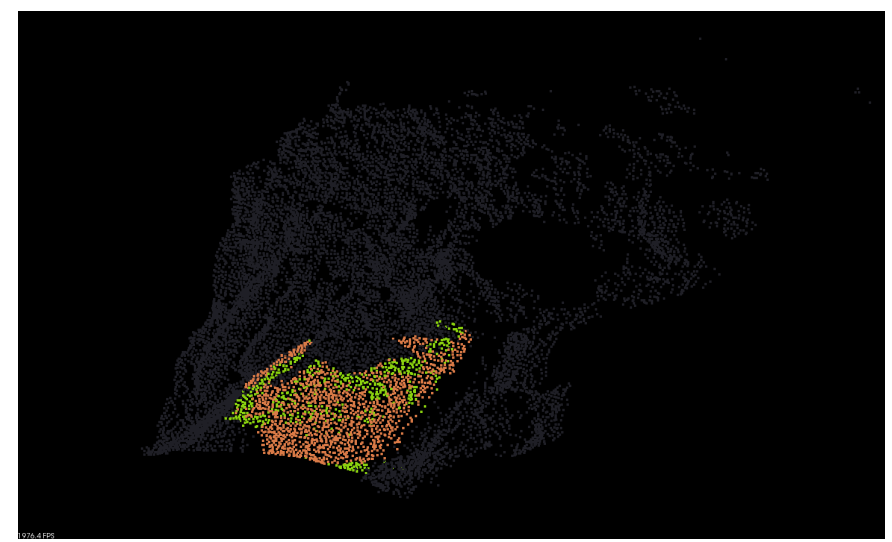

(c) Green+orange: After Step 3.2, Orange: after iterative segmentation; Steps 3.3,3.4.

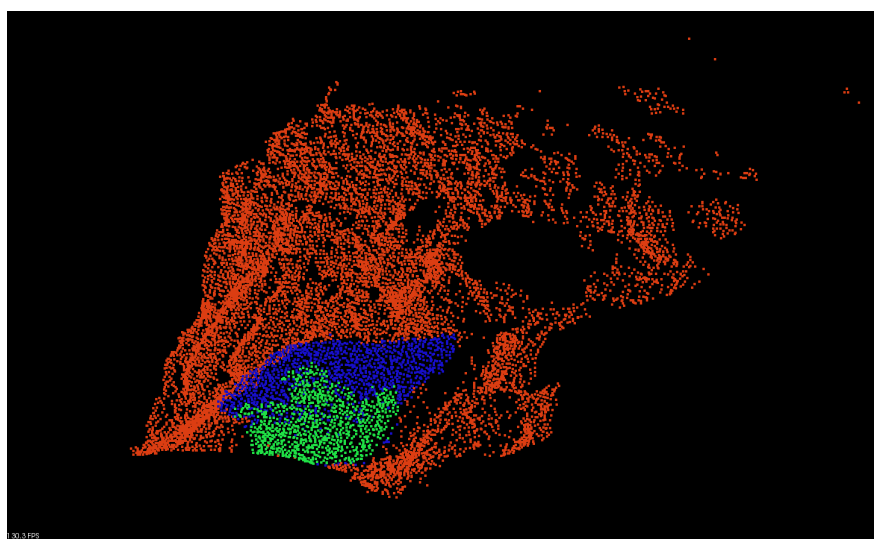

(e) Green: after Step 3.5; blue: added by Step 3.6.

Fig. 3. Step-by-step depiction of the large-object identification algorithm.

makes the density of points approximately uniform over the ranges considered, which is important because beams from the camera spread with distance so the raw data has a much higher density close to the sensor. A minimum size for these voxels should be $d \tan (\delta \theta)$, where $d$ is the distance to the farthest point of interest $(7 \mathrm{~m})$ and $\delta \theta$ is the angular resolution of the sensor $\left(0.24^{\circ}\right)$. Varying density could lead to artefacts in the score since a higher number of points in the same region automatically increases the possible range of scores for the same region. An example of the resulting point cloud is the set of purple points shown in Figure $3 \mathrm{~b}$.

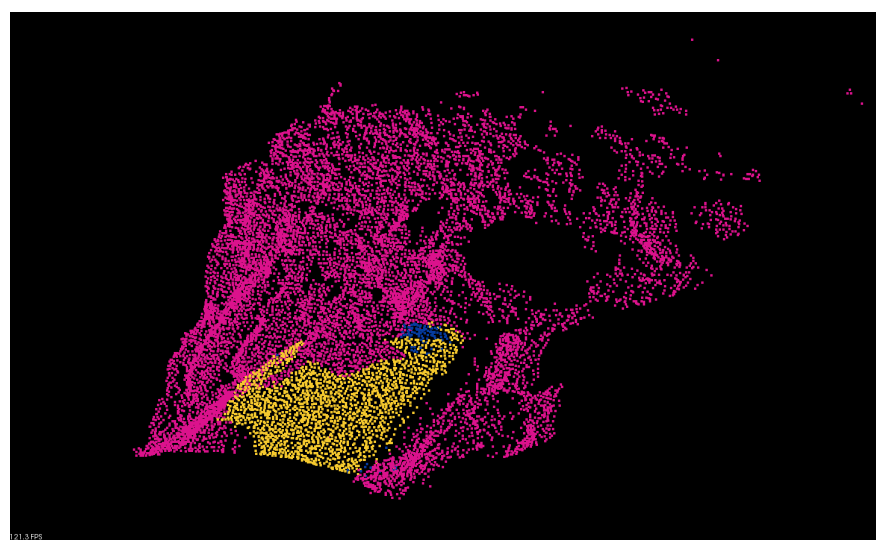

(b) Yellow+blue region: after Step 3.1; blue region: removed by Step 3.2.

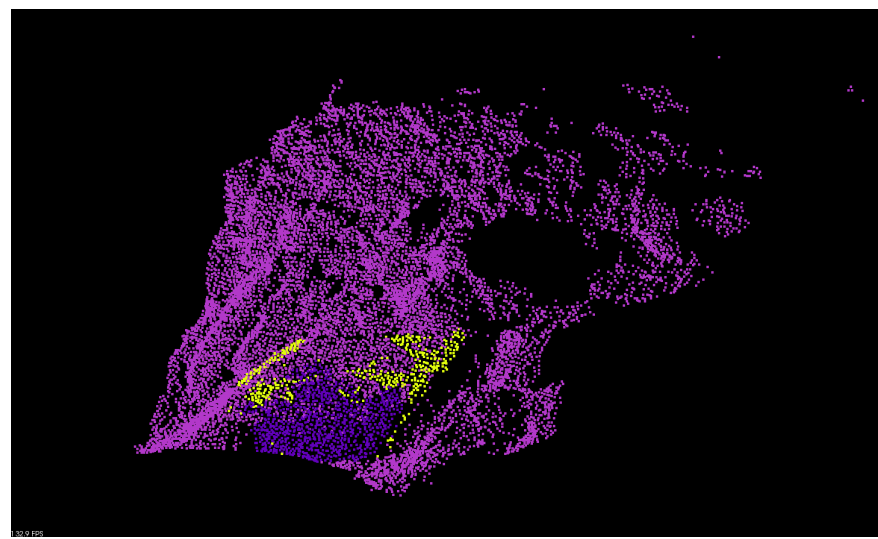

(d) Yellow+dark purple: after iterative segmentation; dark purple: after Step 3.5.

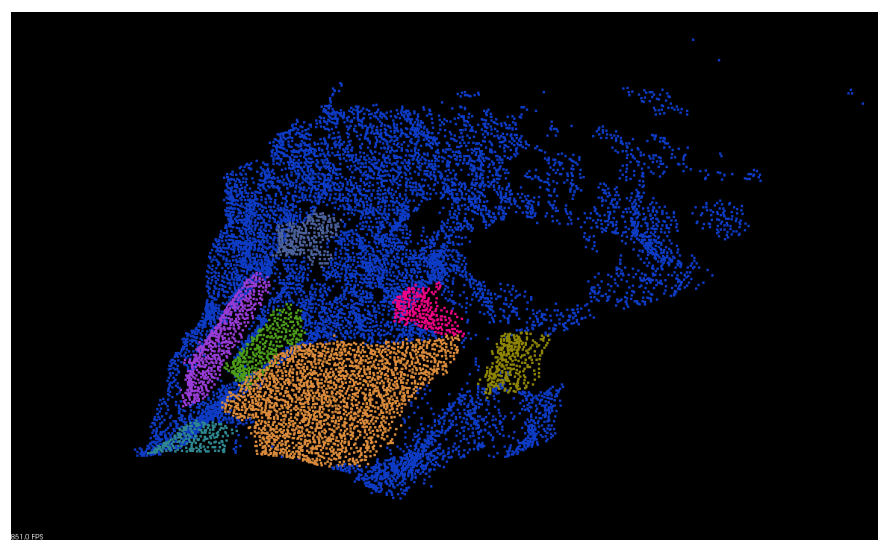

(f) Completed segmentation of all large object candidates.

At this point a $k$-d tree ${ }^{4}$ is set up to efficiently perform frequently used nearest neighbour searches on the current cloud, which is used for all further processing.

\section{Step 2: Surface Characterization}

Points are scored using Algorithm 1, then a standard deviation of scores for the whole pile is computed. One standard deviation is used used as a minimum mean score for a large object candidate to be considered real. Small changes to this

${ }^{4}$ http://pointclouds.org/documentation/tutorials/kdtree_search.php 


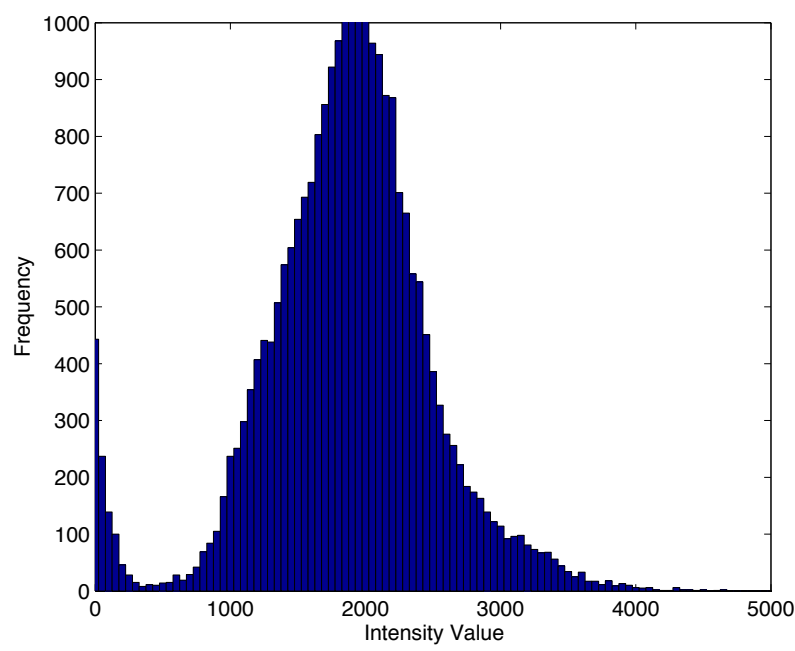

Fig. 4. Filtering data with intensity lower than 500 was found to remove the majority of the noise. Units for intensity were not published with the sensor.

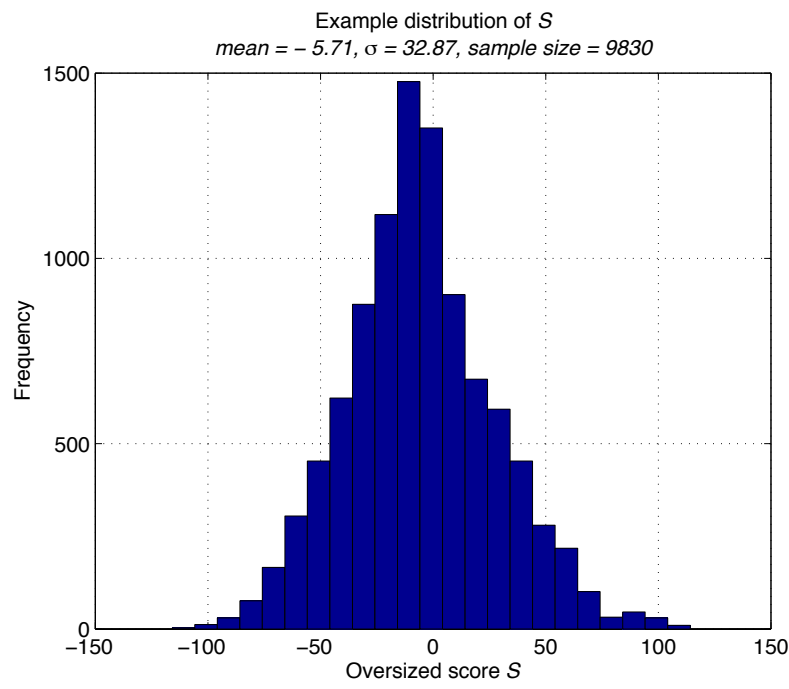

Fig. 5. Typical distribution of oversized score $S$. Small offsets of the mean to the left or right of zero indicate an overall concave or convex shape of the rock pile.

parameter do not have a significant effect on the outcome for real rock piles. This is one way of identifying extrema in a way tuned to the current rock pile, since not all piles are the same. An example of a typical distribution of $S$ is shown in Figure 5. This concept is similar to what is used in [13], where normals associated with the ground plane appear as a peak on a histogram of normal orientations.See, for example, Figure 6.

\section{Step 3: Find Large Objects 1 to $N$}

1) Step 3.1: Extract Region Surrounding Highest Scoring Point: The point with the highest $S$ that has not been flagged is taken as the seed for watershed segmentation with $S$ as the height function. Starting from the seed, a maximum of $n$ jumps of 2.5 times the coarse voxel size are made. This is similar to [3], which used local height maxima to seed regions for

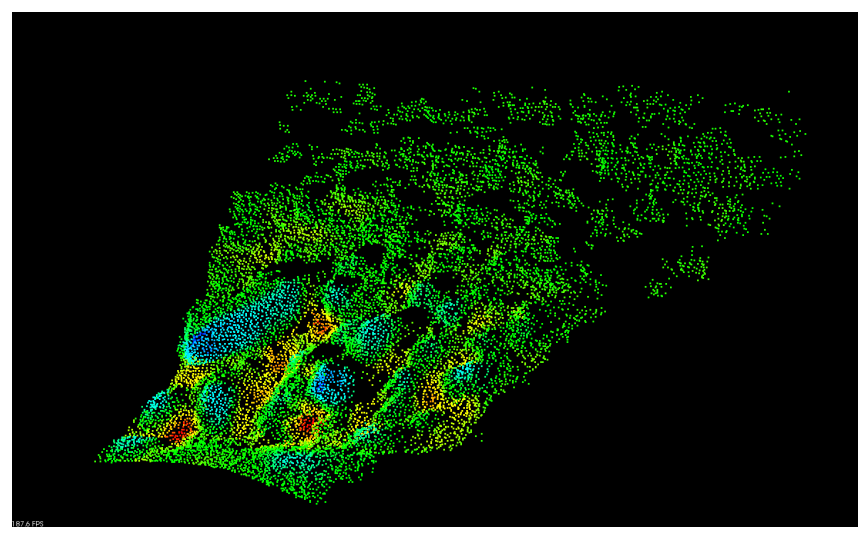

Fig. 6. Results of scoring points using the method described in Algorithm 1 on the point cloud shown in Fig. 7c.

watershed segmentation. Both methods try to start from the peak of the target, but we make less restrictive assumptions about the geometry of the pile. The jump length was sized to allow sparse regions to be traversed while still eliminating isolated clusters of points. An example of the resulting point cloud is shown in Figure $3 \mathrm{~b}$.

Over-segmentation is a common problem with watershed segmentation [21], which was overcome in [22], [23] by merging similar adjacent sections. We take a similar approach, but instead of merging similar regions after segmentation the watershed segmentation overcomes local minima by allowing jumps from one point to another if the destination point has an $S$ exceeding the current point by no more than $10 \%$.

2) Step 3.2: Condition and Separate Main Euclidean Cluster: The next step is to remove rock-pile boundaries by using the same principle as in Algorithm 1, but on a smaller scale. Negatively scoring regions are removed with the purpose of isolating points that make up the large object from a skirt on the rock-pile boundary. Small regions on the surface of the object can sometimes be removed. It may be that some are small enough that they can be recovered in later steps, although we have not verified this. Protection is in place ensure points lying on prominent features $(S \geq 30)$ are kept.

Once boundaries are removed, the central cluster is extracted by using a flood fill algorithm with a simple Euclidean distance criteria starting from the point with the highest $S$. The point cloud size must be above a certain threshold, as in [3], otherwise the segmentation is discontinued and the points surrounding the seed are flagged. An example of the result of this step is shown in Figure $3 b$.

3) Step 3.3: Individual Scoring and Filtering: By this stage, the point cloud is roughly centered on the feature of interest. This means that points farther from the centroid are less likely to be part of a large object. This time, the dot product between the $\mathbf{V}_{c_{j}}$ and $\mathbf{N}_{j}$ becomes the individual score for the point, separate from $S$. The advantage of this is that points scoring negatively that are farther away will pick up a larger negative score; when we remove the lowest scoring fraction of points, which is step Step 3.4 (so long as they score less than zero), points farther away will be eliminated first. This was found to help eliminate points on the rock pile side of boundaries 
refining what was done in Step 3.2. This sequence of scoring points individually and extracting the main cluster is repeated until all points receive an individual score greater than zero or not enough points remain to continue.

This part of the procedure is iterative because describing a convex shape using our method requires a good estimate of its centroid. Refining our estimate of the centroid allows for more reliable identification of points that are part of a convex rock. This procedure is outlined in Algorithm 2. An example of the resulting point cloud is shown in Figure 3c.

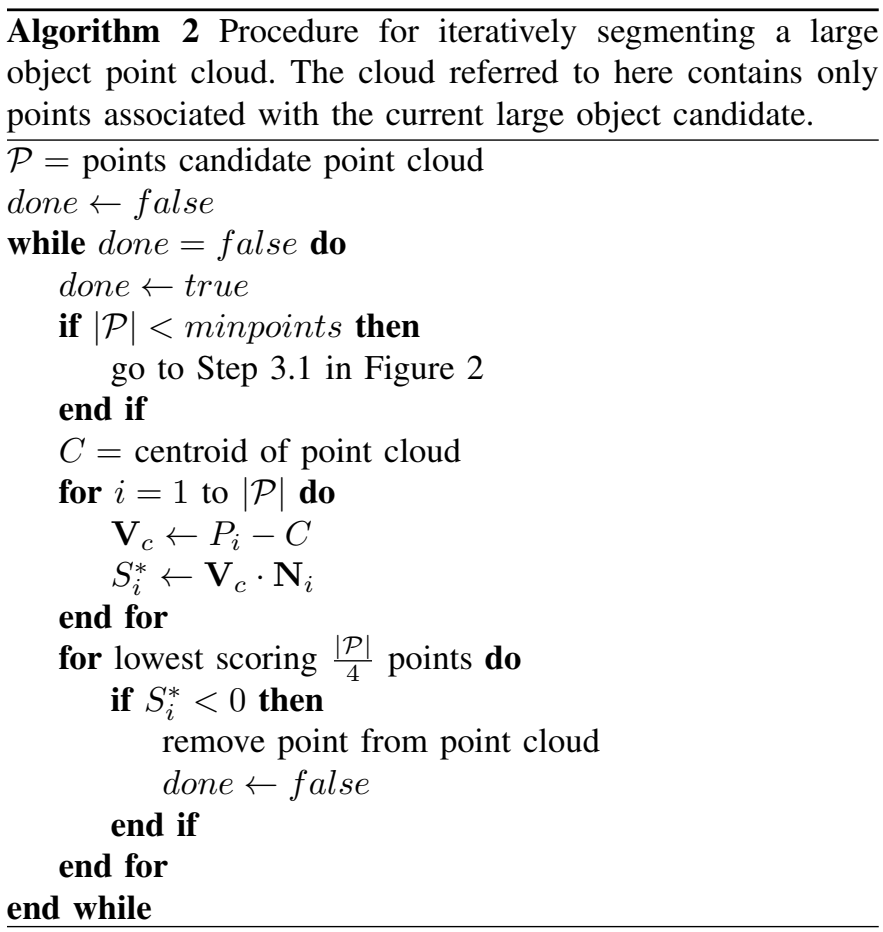

4) Step 3.5: Remove Artefacts: A type of artefact known as a "bridge" was identified in [3] but no specific effort was made to characterize and remove them. Bridges are narrow extensions of the main point cloud that can arise from smaller rocks being present around the base of larger ones. This can happen more frequently when the rock pile is imaged from a large distance (e.g., $>7 \mathrm{~m}$ with our sensor) where noise prevents edges from being resolved accurately.

Let $\mathbf{B}_{c, j}$ denote the vector from $P_{j}$ to the centroid of its neighbors. Neighbors are points within a region larger than the width of the bridges but small enough that the bridge is locally straight. Two measures are used to identify bridges: $\left\|\mathbf{B}_{c, j}\right\|$ and the number of neighbors $m$.

Algorithm 3 presents the algorithm for identifying bridges, where $\mathcal{P}$ is the candidate point cloud, $m$ denotes the number of neighbors for a point, $\bar{m}$ denotes the average number of neighbors for points in $\mathcal{P}$.

The constant $k$ was set to 1.5 for our experiments. Small changes in $k$ do not have a significant effect, however increasing the value of $k$ allows variation in the density of points in the region as well as allowing the bridge to be slightly curved. If $k$ is increased further, points a short distance from the edge (where the number of neighbors are reduced but the $\overline{\text { Algorithm } 3 \text { Procedure for identifying bridges. Only points }}$ part of the large object candidate are considered for this stage.

$$
\begin{aligned}
& \text { for } j=1 \text { to }|\mathcal{P}| \text { do } \\
& \quad \text { if }\left(m_{j}<\bar{m}\right) \&\left(\left\|\mathbf{B}_{c, j}\right\|<k \cdot \operatorname{mean}\left(\left\|\mathbf{B}_{c}\right\|\right)\right) \text { then } \\
& \quad \quad \text { flag point as bridge } \\
& \text { end if } \\
& \text { end for }
\end{aligned}
$$

centroid does not stray far from the center of a region) can be eliminated. As $k$ becomes large, all points that have less than the average number of neighbors are rejected; these would primarily be points along the edge of the point cloud.

A final central cluster extraction is done using a small region around the highest scoring point $S$ as the seed and allowing jumps of $2.5 \times$ (average distance to nearest neighbour). An example of the resulting point cloud is shown in Figure 3d.

5) False-Candidate Identification: An important part of large object identification is to have some measure to determine whether the segmented region actually represents a large object. As mentioned before, the mean $S$ of points representing the object is compared to one $\sigma$ (standard deviation) of $S$ for the whole pile. If the mean score for the object is greater than one $\sigma$ of the pile it is accepted as a significant surface feature of the rock pile and a large object. A hard cut-off could also be used to guard against piles of fine gravel where $\sigma$ can be low (not necessary in our experiments). Finally, the mean score of the whole pile is ignored since we are interested in features on its surface and not the general shape of the rock pile.

6) Step 3.6: Extend Faces: Piles of rock frequently contain rocks with large flat faces much larger than any feature that need to be included as part of the large rock. Watershed segmentation is not well suited to include flat faces [22], therefore we must recover them another way.

For each point in the current representation of the large object, if the average dot product between the normal of each point and the normals of points surrounding it is greater than 0.99 , the region is considered planar. A dot product of 0.99 corresponds to approximately $8^{\circ}$ of float between the normals (of unit length) allowing some tolerance for surface roughness but making it difficult for faces to overflow.

If a point is identified as planar, then points close to it with strongly agreeing normals are added to the point cloud, and the process is repeated until all options are exhausted. An example of the resulting point cloud is shown in Figure 3e. Note that this is done after false object identification because adding flat faces significantly lowers the average score of a point cloud.

Points included in the final large object are flagged in the original point cloud and the process is repeated $N$ times, where $N$ is an upper bound for the number of expected large objects before searching using a separate pre-defined feature size. Ideally, the end result is a number of point clouds representing large objects in the pile which may then be used by a dig planner. If at any stage the point cloud is rejected, the seed region is flagged as used and is not considered to seed another region of the current feature's size. An example of the resulting point clouds is shown in Figure 3f. 


\section{Output Evaluation And Discussion}

This section describes some tests used to evaluate the algorithm on realistic data. Data was gathered at a construction site in Kingston, Canada by capturing point clouds from the TOF camera, which was hand held for most of the trials. In total, 50 point clouds were used to test the algorithm.

To be useful as part of a system for robotic excavation, the system should be capable of performing at least as well as a human operator. A small-scale survey was conducted as a preliminary measure of success. Twelve human subjects were shown color images and raw 3D point clouds side by side (Fig. 7a-d). They were asked to identify large rocks in each image that they would be most concerned with if they were going to use a mining excavator to excavate the pile. Although all the test subjects were not expert machine operators, they were all people who work in mining-related fields (i.e., are familiar with the task).

Each subject was allowed to identify multiple rocks. Rocks identified five times or more are outlined in red on the color photos. Images (a), (c), and (e) from Fig. 7 show that the system successfully identified all rocks that the majority of subjects identified as important. Images (b), (d), and (f) provide examples where large rocks were oriented such that they are difficult for the algorithm to pick out and, as a result, only picked out two of the six rocks commonly identified by users. Looking closely at the points making up rock 2 in Fig. 7, there is significant depth scatter so the points making up the object look more like a cloud as opposed to the points falling on a surface. This lowers their score $S$, making them appear to the algorithm as unlikely to make up a large rock.

Large object 3 is not identified largely due to its orientation relative to the view point. Large portions of its edges are flush with the pile and the edges exposed are on its far side. Since occlusions are not considered, points near far edges like this that end in an occlusion are evaluated as planar sections receiving a low score. Objects 5 and 6 were difficult for both human subjects and the algorithm to spot. This is partially due to range limitations on the sensor. The only way to distinguish rocks such as this in the camera image is to look closely at intensity with a-priori knowledge of the rock's position.

Note the lower intensity of the dirt pile in front of the rocks in Fig. 7(c). A study done by JPL [16] noted that it was best to look at geometric properties when identifying large rocks whereas smaller rocks could be identified using intensity. In some cases the algorithm could be fooled by a mound of dirt with the same shape as a large rock however they might be easily distinguished by looking at intensity. The study done by JPL was for Martian terrain where the sand making up most of the desert reflects light differently to rocks on its surface. In Fig. 7(c) we can see that the sand in the foreground looks significantly different from many rocky features.

\section{CONCLUSions And Future Work}

In summary, this paper describes a new approach for extracting large objects from the irregular surface of a rock pile, as a step towards robotic excavation. Promising experimental results suggest that the approach can perform nearly as well as a human test subject in the task of identifying large or oversize rocks. This is a step towards the long-term objective of creating a dig planner to facilitate robotic excavation in mining that improves efficiency and reduces wear on equipment. Developments in this paper present advantages over previous work in that the individual oversized rocks are segmented.

One avenue for development is to investigate how intensity could be incorporated to address some of the exceptions (unidentified oversize) and improve segmentation. A particular advantage is that this would not require additional information from the sensor. Intensity also shows promise as a way to distinguish between mounds of dirt and large rocks that might otherwise be very similar geometrically.

\section{ACKNOWLEDGMENT}

The authors would like to thank Rob Hewitt for his help with field data collection. This work was supported in part by funding from the Natural Sciences and Engineering Research Council of Canada (NSERC).

\section{REFERENCES}

[1] W. K. W. Cheung, "Inferring surface structure of rock piles from range images," Master's thesis, McGill University, Department of Mining and Metallurgical Engineering, Montreal, Canada, June 1992.

[2] A. Bedair, "Digital image analysis of rock fragmentation from blasting," Ph.D. dissertation, McGill University, Montreal, Canada, September 1996.

[3] M. J. Thurley, "Three dimensional data analysis for the seperation and sizing of rock piles in mining," Ph.D. dissertation, Monash University, Department of Electrical and Computer Systems Engineering, December 2002.

[4] J. Seo, S. Lee, J. Kim, and S. Kim, "Task planner design for an automated excavation system," Automation in Construction, vol. 20, no. 17, pp. 954-966, November 2011.

[5] J. A. Marshall, T. D. Barfoot, and J. Larsson, "Autonomous underground tramming for center-articulated vehicles," Journal of Field Robotics, vol. 25 , no. 6-7, pp. 400-421, Jun. 2008.

[6] M. Magnusson and H. Almqvist, "Consistent pile-shape quantification for autonomous wheel loaders," in Proceedings of the 2011 IEEE/RSJ International Conference on Intelligent Robots and Systems, September 2011, pp. 4078-4083.

[7] J. Marshall, P. Murphy, and L. Daneshmend, "Toward autonomous excavation of fragmented rock: Full-scale experiments," IEEE Transactions on Automation Science and Engineering, vol. 5, no. 3, pp. 562-566, Jul. 2008.

[8] D. A. Bradley and D. W. Seward, "Developing real-time autonomous excavation-the LUCIE story," in Proceedings of the 34th IEEE Conference on Decision and Control, vol. 3, no. December, 1995, pp. 30283033.

[9] S. Sarata, N. Koyachi, and K. Sugawara, "Field test of autonomous loading operation by wheel loader," in Proceedings of the 2008 IEEE/RSJ International Conference on Intelligent Robots and Systems, Nice, France, September 2008.

[10] H. Takahashi and K. Sano, "Automatic detection and breaking system for boulders by use of ccd camera and laser pointer," Fragblast, vol. 2, no. 4, pp. 397-414, Jan. 1998.

[11] M. Petty, "Autonomous LHD loading," in Proceedings of the Fourth Annual Conference on Mechatronics and Michine Vision in Practice, September 1997, pp. 219-224.

[12] M. Magnusson, "The three-dimensional normal-distributions transform - an efficient representation for registration, surface analysis, and loop detection," Ph.D. dissertation, Örebro University, Örebro, Sweden, 2009.

[13] S. Betge-Brezetz, R. Chatila, and M. Devy, "Natural scene understanding for mobile robot navigation," in Proceedings of the 1994 IEEE International Conference on Robotics and Automation, vol. 1, May 1994, pp. 730-736.

[14] V. Gor, R. Castano, R. Manduchi, R. Anderson, and E. Mjolsness, "Autonomous rock detection for mars terrain," Jet Propulsion Laboratory, California Institute of Technology, Tech. Rep., 2001. 


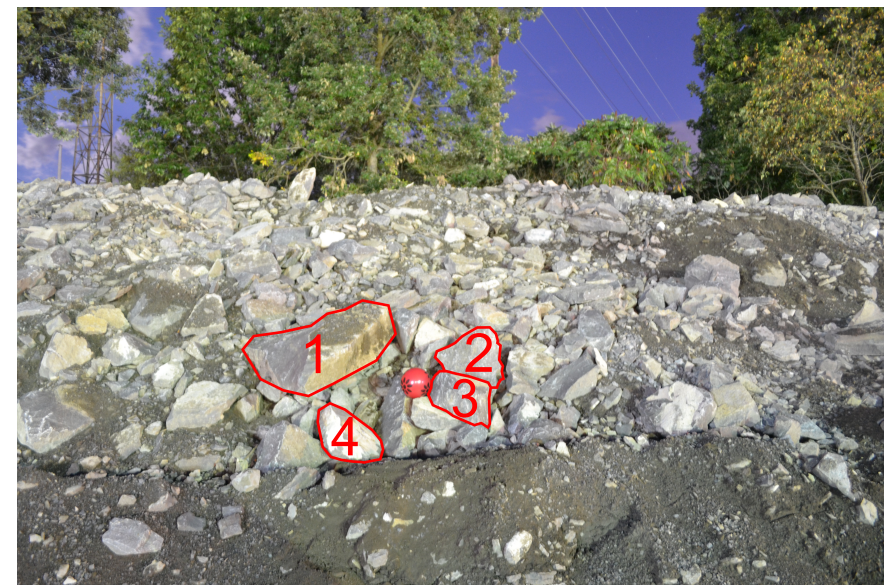

(a)

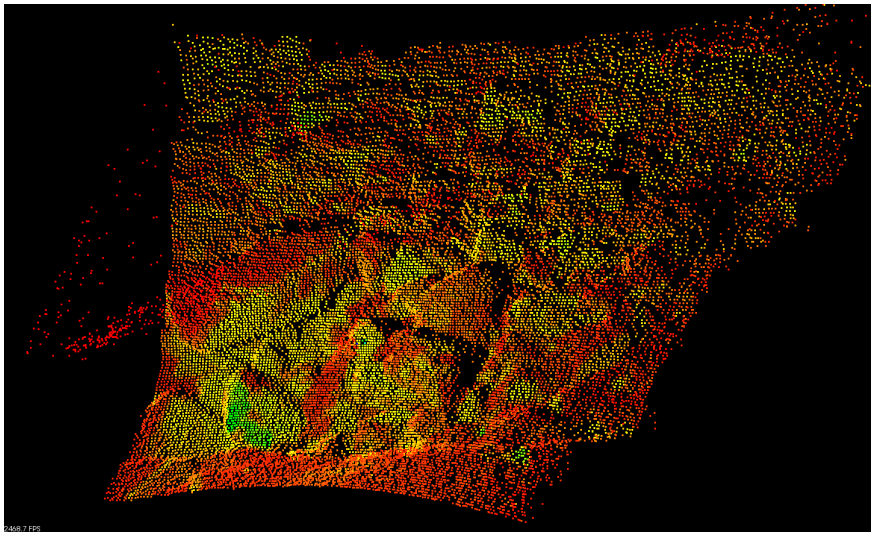

(c)

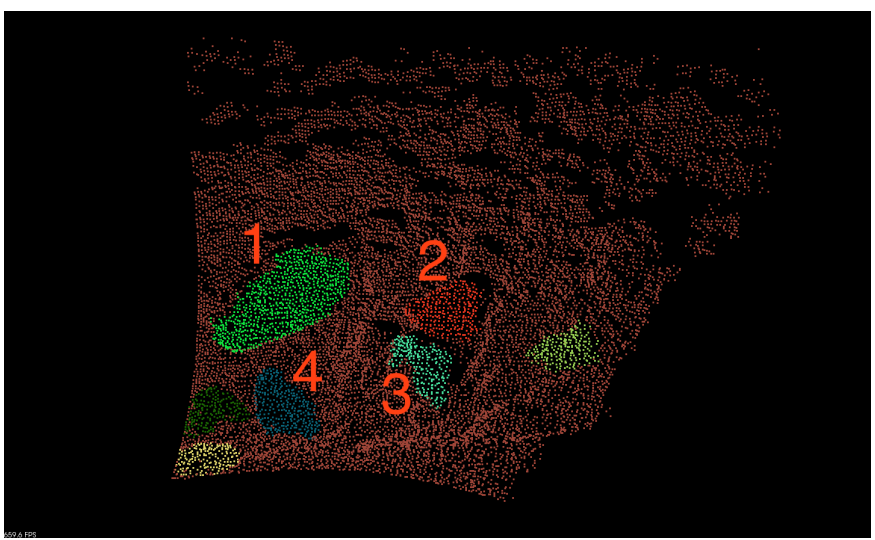

(e)

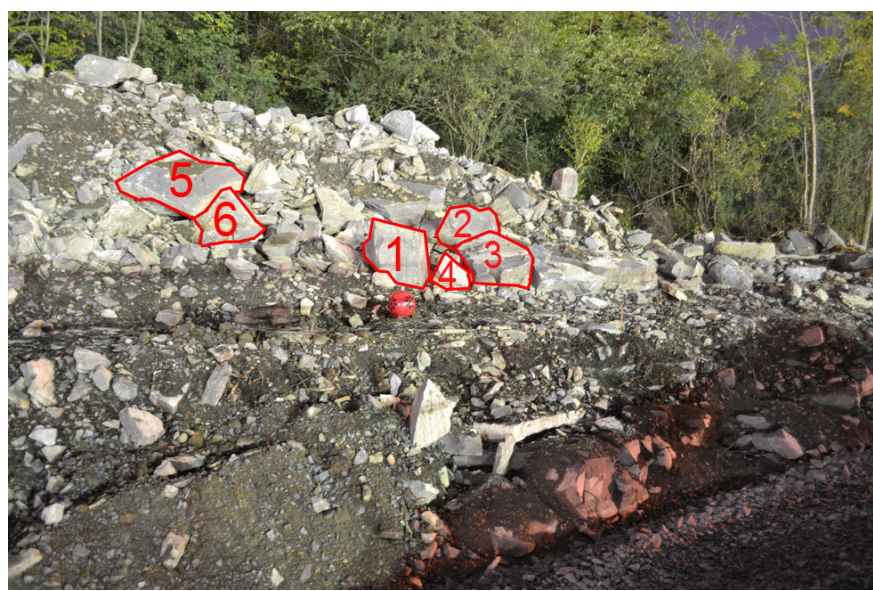

(b)

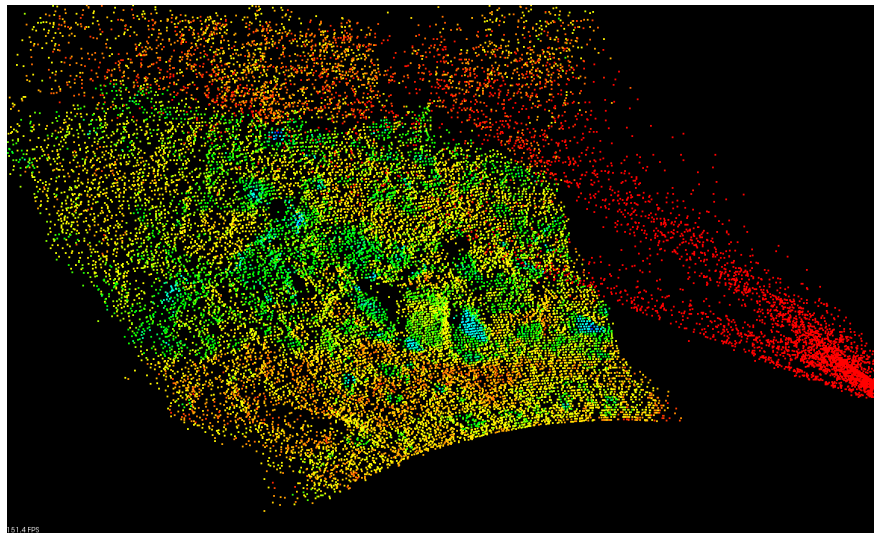

(d)

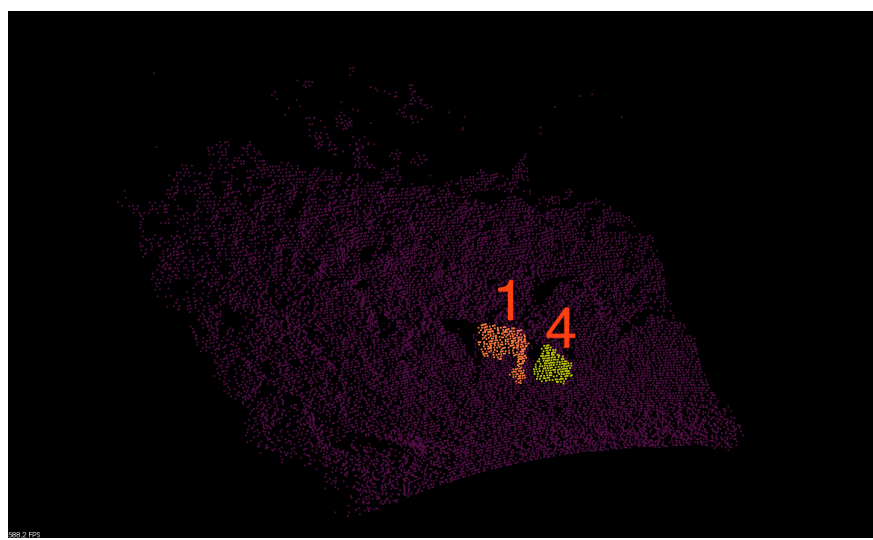

(f)

Fig. 7. Images of point clouds used for user survey. Parameters were not adjusted between (e) and (f). Note that the LiDAR field of view only covers from just below the red ball to just above rock five and the left and right boundaries are just to the right of rock 3 and just to the left of rock 5 in the top images.

[15] T. Choi, H. Delingette, M. Deluise, Y. Hsin, M. Herbert, and K. Ikeuchi, "A perception and maniuplation system for collecting rock samples," The Robotics Institute, Carnegie Mellon University, Pittsburgh, PA, Tech. Rep., 1990.

[16] J. Fox, R. Castano, and R. C. Anderson, "Onboard autonomous rock shape analysis for mars rovers," in Proceedings of the 2002 IEEE Aerospace Conference, vol. 5, March 2002.

[17] H. Ng, S. Ong, K. Foong, P. Goh, and W. Nowinski, "Medical image segmentation using k-means clustering and improved watershed algorithm," in Proceedings of the 2006 IEEE Southwest Symposium on Image Analysis and Interpretation, 2006, pp. 61-65.

[18] M. J. Thurley, "Automated online measurement of limestone particle size distributions using 3D range data," Journal of Process Control, vol. 21 no. 2, pp. 254-262, 2011.

[19] — "Automated image segmentation and analysis of rock piles in an open-pit mine," in Proceedings of the 2013 International Conference on Digital Image Computing: Techniques and Applications, 2013, pp. 1-8.

[20] B. Büttgen and P. Seitz, "Robust optical time-of-flight range imaging based on smart pixel structures," IEEE Transactions on Circuits and Systems, vol. 55, no. 6, pp. 1512-1525, 2008.

[21] W. Bieniecki, "Oversegmentation avoidance in watershed-based algorithms for color images," in Proceedings of the International Conference on Modern Problems of Radio Engineering, Telecommunications and Computer Science, February 2004.

[22] A. Mangan and R. Whitaker, "Partitioning 3d surface meshes using watershed segmentation," IEEE Transactions on Visualization and Com- 
puter Graphics, vol. 5, no. 4, pp. 308-321, 1999.

[23] J. Jiang, Z. Zhang, and Y. Ming, "Data segmentation for geometric feature extraction from Lidar point clouds," in Proceedings of the 2005 IEEE International Geoscience and Remote Sensing Symposium, vol. 5 , July 2005, pp. 3277-3280. 